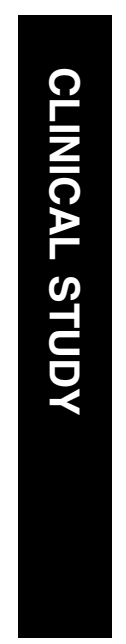

\section{Ocular air gun injuries: a one-year surveillance study in the UK and Eire (BOSU). 2001-2002.}

${ }^{1}$ Department of Ophthalmology, Singleton Hospital, Swansea, West Glamorgan, UK

${ }^{2}$ Department of Ophthalmology, St James' University Hospital, Beckett Street, Leeds, UK

${ }^{3}$ Department of Ophthalmology, Bristol Eye Hospital, Lower Maudlin Street, Bristol, UK

${ }^{4}$ International Centre for Eye Health, London School of Hygiene and Tropical Medicine, Keppel Street, London, UK

\section{${ }^{5}$ Department of} Ophthalmology, University Hospital South Wales, Cardiff, UK

Correspondence: GN Shuttleworth, Department of Ophthalmology, Singleton Hospital, Sketty Lane, Swansea, West Glamorgan SA2 8QA, UK

Tel: + 00441792205666 Ext 6709.

E-mail: garryshuttleworth@ hotmail.co.uk

Received: 21 April 2008 Accepted in revised form: 14 August 2008 Published online: 19 September 2008

\begin{abstract}
Aims The vulnerability of the eye means that ocular air gun injuries figure prominently in the medical literature. This Study reports the results of the first ocular air gun injury surveillance study.

Methods Ocular air gun injuries were reported to the British Ophthalmic Surveillance Unit (BOSU; United Kingdom and Eire) for the period November 2001-December 2002 (13 months). Two questionnaires were used to collect demographic details, circumstances of injury, details of injuries, medical management and outcome.

Results A total of 105 initial and 99 follow-up questionnaires were returned. Eighty-six ocular air gun injuries occurred during the last 12 months of surveillance yielding a corrected, estimated incidence of 91-115 injuries/year. Injuries were most frequent in August/September, and 90\% (95/105) of victims were men with mean age of 17.5 years ( $74 \%$ under 18 years). In all, $40 \%$ $(32 / 81)$ of injuries occurred at home and $53 \%$ $(43 / 81)$ in a public place. $23 \%(19 / 84)$ of injuries were deliberate, $66 \%(69 / 104)$ of injuries were severe and $20 \%(21 / 105)$ resulted in ruptured globes. In all, 54\% (48/89) required hospital admission and 41 required surgery. A total of $11 \%(12 / 105)$ of eyes were either enucleated or eviscerated. Final visual acuity was $\leqslant$ counting fingers in $29 \%(26 / 91)$ but $>6 / 12$ (Snellen) in 65\% (59/91). Moderate/ significant cosmetic deformities were recorded in $10 \%(8 / 77)$ and restricted ocular movements in $5 \%(4 / 72)$.

Conclusions Ocular air gun injuries damage sight and leave lasting morbidity. The demographics and circumstances of injury are well documented with access to, and unsupervised use of, air
\end{abstract}

GN Shuttleworth', P Galloway2 , JM Sparrow ${ }^{3,4}$ and C Lane ${ }^{5}$ guns, appearing the principal risks for injury.

Eye (2009) 23, 1370-1376; doi:10.1038/eye.2008.275; published online 19 September 2008

Keywords: air guns; orbit; injury; trauma

\section{Introduction}

There are a wide variety of air guns available in the United Kingdom including pop guns, pellet guns (spud guns), BB (ball bearing) guns, soft air guns, paintball guns, and 0.177 , and 0.22 calibre air pistols and rifles, in all of which projectiles are forced from the barrel by compressed air or gas. It is estimated that there may be as many as $4-4.5$ million air guns in the United Kingdom. ${ }^{1}$

Case reports and extended series of air gun shootings are not infrequent in the medical literature with reports of lifelong disability and fatalities. Although the Home Office publishes annual Crime Figures for England and Wales, including those involving air guns, these figures only reflect crimes of which the police are aware. Robust epidemiological data relating to such injuries is lacking.

To gather data for an accurate estimate of the incidence of air gun injuries, a national surveillance mechanism is needed. The British Ophthalmic Surveillance Unit (BOSU) offers a mechanism by which, at least, ocular air gun injuries can be reported.

This study, which is the first air gun surveillance study of its kind, was designed to determine the incidence of ocular air gun injuries, and to describe the circumstances of injury, the injuries sustained, medical interventions, and lasting effects. The data collected attempts to quantify the hazard to guide future preventative strategy. 


\section{Methods}

For 13 months, from November 2001 to December 2002, the yellow BOSU surveillance card, sent to all permanently employed ophthalmologists in the United Kingdom and Eire, included a category for ocular air gun injury.

Case inclusion was defined as any individual, any age, sustaining any ocular, orbital, or adnexal injury resulting from the use/misuse of any air gun (BB gun/air pistol/ air rifle/or air gun).

Study objectives were to determine:

(1) the incidence of ocular air gun injuries.

(2) the circumstances of injury.

(3) the demographic characteristics of those involved.

(4) the ocular injuries sustained.

(5) the medical interventions performed.

(6) lasting morbidity.

Recipient ophthalmologists of the BOSU surveillance card were asked to return the card indicating whether they had seen any ocular air gun injury in the preceding month. The card was then returned to BOSU and the study investigator was informed.

Reporting ophthalmologists were then sent two questionnaires, each with an explanatory key to aid the completion. Both questionnaires were designed to be face valid and easy to complete. The first questionnaire was sent immediately and the second follow-up questionnaire was sent 6 months later. Where questionnaires were not returned within 3 months, a further questionnaire was sent to the reporting ophthalmologist. Queries were handled by correspondence on a case-by-case basis.

The first questionnaire gathered demographic information, and information relating to the injury and its immediate management, whereas the follow-up questionnaire gathered information detailing subsequent management and lasting effects (Table 1).
Data from the returned questionnaires were transferred to a database before being analysed using Microsoft Excel and SPSS software.

For the purpose of analysis, Snellen acuities were ranked for non-parametric analysis and converted to the $\log _{10}$ of the Snellen fraction (equivalent of logMAR) for parametric analysis. The scale was extended assuming 'count fingers' equivalent to 1.6 logMAR (Snellen 1.5/60), 'hand movement' equivalent to $1.9 \log$ MAR (Snellen $0.75 / 60)$, 'perception of light' equivalent to $2.2 \log$ MAR (Snellen 0.375/60), ${ }^{2}$ and 'no perception of light' equivalent to $3 \log$ MAR (Snellen 1/6000).

In view of the questionnaire design and the surveyed respondent population, where there was no marked response to a question it was assumed that the response was 'other/unknown'. As there is no reason to suppose a systematic or reporting bias, missing data were assumed to be randomly distributed. Denominators vary depending upon missing data and question type.

\section{Results}

\section{Incidence of ocular injuries}

During the 13-month surveillance, 145 separate notifications were received. Of these, 20 responders failed to reply to any correspondence, four were false positives (mistakes), seven could not recall the victim's name, three were reported outside the study interval (one before and two after), and six were duplicates. The overall response rate from ophthalmologists receiving the yellow BOSU card for the surveillance period was $76 \%$.

Analysis was, therefore, based upon 105 immediate and 99 follow-up questionnaires. A high response during the first month of surveillance represents a first pass phenomenon whereby injuries occurring within the preceding few months are reported simultaneously.

In the subsequent 12 months of surveillance, data relating to 86 air weapon injuries were collected. This

Table 1 Questionnaire information collection

\begin{tabular}{|c|c|c|}
\hline \multicolumn{2}{|c|}{ First questionnaire } & \multirow{2}{*}{$\begin{array}{l}\text { Follow-up questionnaire } \\
\text { Management, outcome, and lasting effects }\end{array}$} \\
\hline Demographic information & $\begin{array}{l}\text { Injury and immediate } \\
\text { management }\end{array}$ & \\
\hline Sex & Side of injury & Number and type of medical interventions \\
\hline Date of birth of victim & Visual acuity at presentation & Final visual acuity and cause of visual loss \\
\hline Date of injury & Any previous ocular history & Ocular motility of injured eye \\
\hline Where and how the injury occurred & Location of the pellet & Number of in-patient days \\
\hline Type and calibre of air gun & Details of imaging performed & Number of outpatient attendances \\
\hline Projectile shape and material & Initial medical management and interventions & \\
\hline
\end{tabular}


figure represents the best estimate of the absolute minimum incidence.

Eighty-seven injuries were reported from England, nine from Scotland, five from Wales, three from Northern Ireland, and one from Eire. The frequency of injury appeared to peak in August (14 injuries) and September (12 injuries).

\section{Demographics and injuries}

The mean age of victims was 17.5 years (SD 9.12, range $5-78)$ with $74 \%(78 / 105)$ being $<18$ years old and $90 \%$ (95/105) being male. Fifty-six right and 49 left eyes were involved.

In all, $0.177 / 0.22$ calibre air guns were identifiable where stated or where they could be inferred - where the material and shape of the projectile indicated a metal diabolo. In all 19\% (20/105), 0.177/0.22 air gun injuries and $51 \%$ (54/105) BB gun injuries were definitively identified. In addition, one soft air gun, one plastic toy gun, and a paintball gun were identified. In 28 cases, it was not possible to categorise accurately the type of air gun involved.

Thirty-two injuries occurred at home, 43 in a public place, and two occurred at school; 65 were accidents, but 19 were deliberate shootings. Where known, 65\% (54/83) were inflicted by friends or relatives and $12 \%(10 / 83)$ were self-inflicted accidents. Information relating to supervision was available in only 41 of the 78 injuries occurred to persons under 18 years of age, where a supervising adult was reportedly present in just five cases.

The resulting ocular injuries were categorised into severe injuries 66\% (69/104), penetrating/perforating injuries/globe ruptures 20\% (21/104), and 'minor' injuries 34\% (35/104).

\section{Medical management}

Excluding two outliers with very delayed presentations, the vast majority of victims presented immediately following their injury (mean 0.31 days, SD 0.79 , range $0-4)$. Fifty-four percentage (48/89) of victims were admitted to hospital and follow-up ranged from 5 to 457 days (mean 147, median 120, $n=89$ ). Total in-patient stay in hospital averaged 2.5 days (SD 3.54, $n=89$ ) and ranged from 0 to 18 days.

A total of 34 projectiles were retained within the tissues of the head and neck of which eight were intraocular and two intracranial. In all, 94 imaging investigations were performed in 66 cases, including 24 CT scans and one MRI. Ultrasound was performed in 44 cases and X-rays in 25.
Forty-one victims required surgery, 26 within 2 days of the injury. Thirteen required two operations, and four needed three operations. Of the 62 procedures performed, 57 were under general anaesthesia. Eight eyes were enucleated and another four eviscerated. Four enucleations (three with implants) plus one evisceration with implant, were performed as primary procedures. At the conclusion of the data collection, four patients were awaiting further surgery.

Data relating to pharmacological treatments were incomplete restricting meaningful analysis. However, predictably antibiotics, steroids, and cycloplegic medications formed the mainstay of treatment, supplemented with ocular hypotensive medication, analgesics, and NSAIDS.

\section{Visual function}

Visual acuity at presentation was $<6 / 60$ in 50\% (50/101) of cases and no light perception in 13 cases (logMAR mean 1.23, median 1.00; Figure 1; Table 2). Three victims (2.9\%) had a previous significant ipsilateral ocular history of amblyopia or squint, in accordance with the population incidence of amblyopia. ${ }^{3,4}$

Final visual acuity was recorded $<6 / 60$ in $29 \%(26 / 91)$ of cases (no light perception in 19 cases) and $>6 / 12$ in 65\% (59/91; logMAR mean 0.81, median 0.00; Figure 1). Although visual acuity improved in $60 \%(53 / 88)$ cases, it remained unchanged in $27 \%(24 / 88)$ and deteriorated in $13 \%(11 / 88 ; \Delta \log \mathrm{MAR}=0.45 ; P<0.05)$.

The cause of visual loss included corneal damage, glaucoma, macula hole, retinal detachment, optic nerve injury, phthisis, and lost globes. The cosmetic and oculomotor effects of the injuries are summarised in Table 3.

\section{Type of air gun}

Presenting visual acuity was poorer in $0.177 / 0.22$ calibre air gun injures than BB gun injuries (mean 1.79 logMAR, median 1.90, compared to mean $1.00(P<0.05)$, median $0.78(P<0.05)$, and visual acuity $<6 / 60$ in $72 \%(13 / 18)$ compared to $39 \%(21 / 54(P<0.05))$.

Final visual acuity improved less frequently (40\% (6/15) compared to $73 \%(35 / 48) P>0.05)$ and to a lesser degree (0.12 compared to 0.67 LogMAR; $P<0.05)$ in $0.177 / 0.22$ calibre air gun injuries compared to $\mathrm{BB}$ gun injuries. The change between presenting and final visual acuity for $0.177 / 0.22$ calibre air gun injuries did not in itself reach statistical significance.

Final visual acuity was <6/60 in 59\% (10/17) of $0.177 /$ 0.22 calibre air gun injuries as compared to $12 \%(6 / 49)$ of BB gun injuries $(P<0.05)$. Final visual acuity was $\geqslant 6 / 12$ 
in only $24 \%(4 / 17)$ of $0.177 / 0.22$ calibre air gun injuries compared with $86 \%(42 / 49)$ of BB gun injuries $(P<0.05)$.

Although severe injuries were common with $\mathrm{BB}$ guns $(69 \%)$, injuries resulting in penetration/perforation/ globe rupture were less frequent than with $0.177 / 0.22$ calibre air guns (7\% (4/54) compared with $30 \%(6 / 20)$; $P<0.05)$. Retained projectiles were reported in $75 \%$ $(15 / 20)$ of $0.177 / 0.22$ calibre air gun injuries and $20 \%$ $(6 / 30)$ of BB gun injuries (including two intraocular) $(P<0.05)$. Correspondingly, surgical intervention was required in $85 \%(17 / 20)$ of $0.177 / 0.22$ calibre air gun
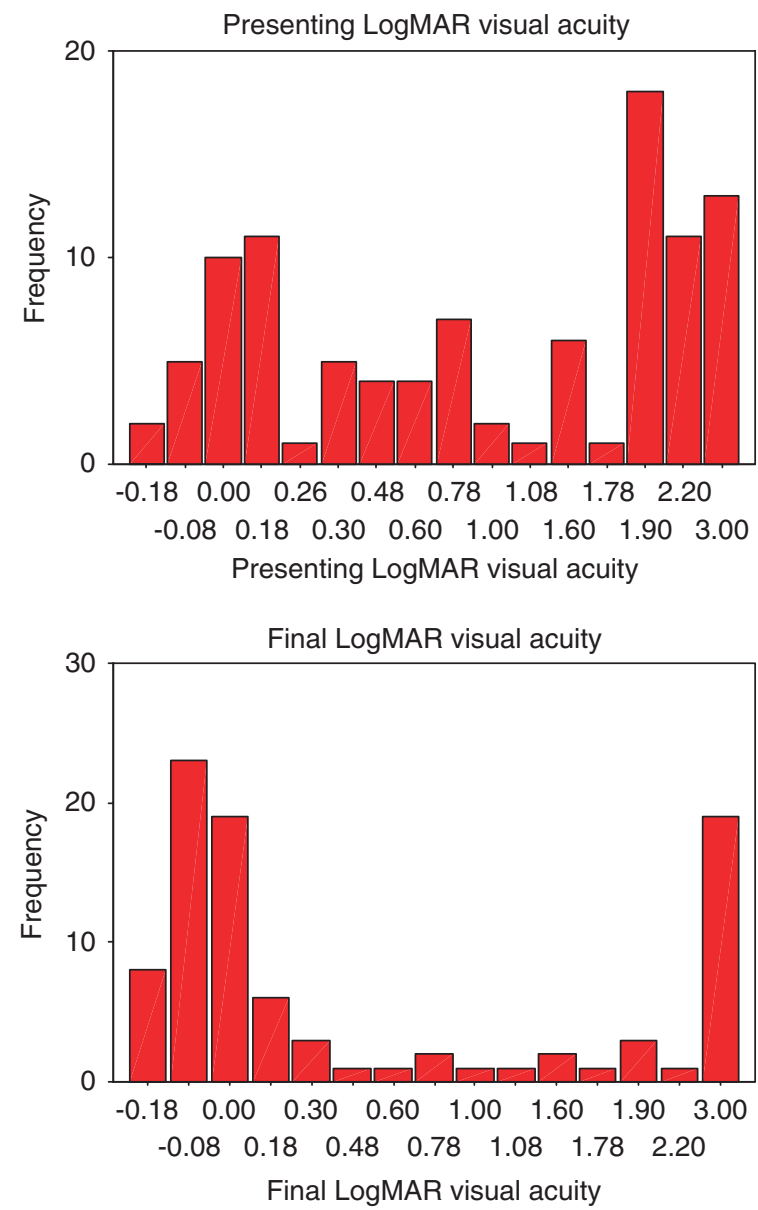

Figure 1 Histogram of visual acuity at presentation and final visit. Count fingers equivalent to $1.6 \log \mathrm{MAR}$, hand movement equivalent to $1.9 \log M A R$, perception of light equivalent to $2.2 \log \mathrm{MAR}$, and no perception of light equivalent to $3 \log \mathrm{MAR}$. injuries, but only $24 \%(13 / 54)$ of BB gun injuries $(P<0.05)$. Victims of $0.177 / 0.22$ calibre air guns spent an average, 5 days in hospital compared to less than 2 days for the victims of BB gun injuries $(P<0.05)$.

\section{Accidental and deliberate injuries}

Victims of deliberate shootings were older than victims of accidental shootings (mean age 21.8 years compared to 16.7 years; $P<0.05)$. Deliberate shootings were also more frequently the result of $0.177 / 0.22$ calibre air guns $(62 \%$ $(8 / 13)$ compared to $20 \%$ (10/49) for BB guns; $P<0.05)$, and were more likely to occur in a public place than accidental shootings (83\% (15/18) compared to $34 \%$ $(17 / 50) P<0.05)$. Victims with a final visual acuity of $<6 / 60$ were more likely to have been shot by a $0.177 / 0.22$ calibre air gun than a BB gun than those with a final visual acuity of $6 / 12$ or better $(63 \%(10 / 16)$ compared to $9 \%(4 / 46) P<0.05)$.

\section{Presenting visual acuity}

Visual acuity, at presentation, of $<6 / 60$ was more frequently associated with severe injuries (86\% (42/49) compared to $53 \%(27 / 51) ; P<0.05)$, penetrating/ perforating/globe ruptures $(42 \%(21 / 50)$ compared to $0 \%$ $(0 / 51) ; P<0.05)$, retained projectiles $(67 \%(24 / 36)$ compared to $19 \%(6 / 32) P<0.05)$, and the need for surgical intervention (64\% (32/50) compared to $14 \%(7 / 51)$; $P<0.05)$. All enucleations and eviscerations occurred in eyes with a presenting visual acuity of $<6 / 60$.

Final visual acuity was $<6 / 60$ in $60 \%(26 / 43)$ and $6 / 12$ or better in $38 \%(16 / 43)$ of those with a presenting visual acuity worse than $6 / 60$ compared to $2 \%(1 / 46$;

Table 3 Effects on ocular movement and cosmetic appearance

\begin{tabular}{lcr}
\hline Effects on ocular movements & Number & $\%$ \\
\hline Normal & 72 & 94.7 \\
Restricted & 4 & 5.3 \\
& & \\
Cosmetic appearance & 69 & 89.6 \\
$\quad$ Unaffected/slight & 4 & 5.2 \\
Moderate deformity & 4 & 5.2 \\
Significant deformity & & \\
\hline
\end{tabular}

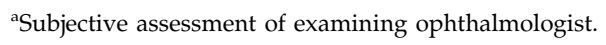

Table 2 Presentation and final visit visual acuities

\begin{tabular}{lcccrr}
\hline Visual acuity $(\log M A R)$ & Mean & SD & Range & Number & P \\
\hline Presentation & 1.23 & 1.05 & -0.18 & NPL & 101 \\
Final visit & 0.81 & 1.25 & -0.18 & NPL & 91 \\
Change in visual acuity (logMAR) & 0.45 & 0.82 & -1.12 & NPL & 88 \\
\hline
\end{tabular}


$P<0.05)$, and $93 \%(43 / 46 ; P<0.05)$ for those with a better presenting acuity.

\section{Discussion}

There are numerous reports of air gun injuries and deaths recounted in the medical literature. The vulnerability of the eye and the profound effects upon vision mean that ocular injures figure prominently. Most of them report broadly similar findings (Table 4) with variations in outcome probably attributable to the method of case identification. This study represents the only prospective surveillance study of air gun injuries, and currently provides the best estimate of the true incidence and ocular morbidity associated with air gun injuries in the United Kingdom and Eire.

Omission of the injuries reported during the first month of surveillance (first pass bias) yields an estimated annual incidence of 86 air gun injuries, a figure, which should probably be regarded as a best estimate of the minimum incidence of such injuries. The lack of alternative sources for recording or detecting air gun injuries meant that formal capture-recapture methodology and study-specific ascertainment rates could not be calculated. However, estimates from the previous BOSU studies and surveys place case ascertainment rates between 75 and $94 \% .{ }^{17}$ If these rates are representative, then the true 12-month incidence rate for ocular air gun injuries would be between 91 and 115 cases. In reality, however, robust methodology, unambiguous case definition, and no reason to suspect a systematic reporting bias, mean that the case ascertainment is likely to be high. Although this study was limited to the reporting of ocular injuries, previous studies have suggested that one-third of all air gun injuries involve the head and neck, let alone the eye $\mathrm{e}^{18}$ implying that the total number of air weapon injuries in the United Kingdom and Eire each year is probably at least 3 times the incidence of the ocular injuries reported.

Air gun crime appears to be increasing with 13756 air gun crimes reported in 2003/4. Of these, 3079 were of crimes of violence against the person including in 138 cases actual or attempted homicide. 2395 air gun crimes were reported as causing injuries, 157 of which were categorised as serious. Although no fatal injuries were reported for 2003/4, four fatalities were reported in the previous 3 years. ${ }^{19}$ Where known, $77 \%$ of the injuries reported in this study were the result of accidents. It is reasonable to assume that the majority of these would not be reported to the police, and therefore would not figure prominently in crime statistics. This assumption is born out by the fact that victims of recorded crime tend to be significantly older than those injured in this study.

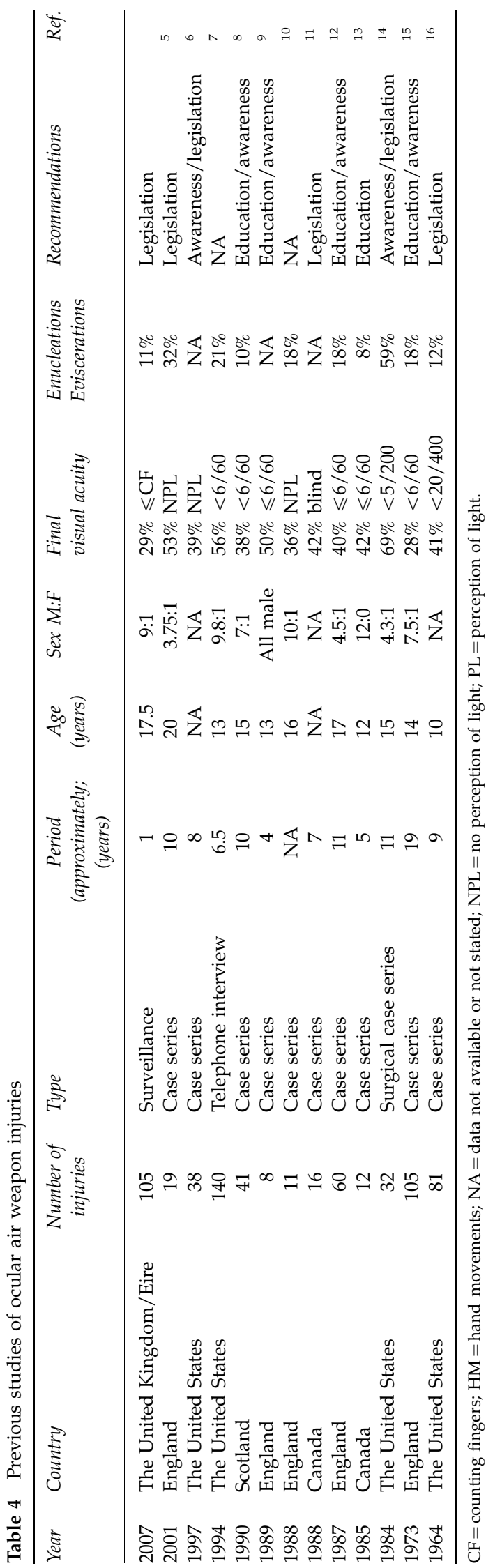


It is probable that a little over half of the injuries reported in this study would have been recorded by Home Office criteria. ${ }^{20}$ Home Office statistics, therefore, represent a significant underestimate of the true annual air gun morbidity.

Althought the numbers are relatively small, the peak incidence for the ocular injuries reported occurred at the end of the summer/early autumn perhaps suggestive of favourable weather and bored youngsters at the end of their summer recess. The demographic characteristics of the victims in this surveillance study are similar to those reported in previous series. Almost three quarters of victims were under 18 years of age, male victims out numbered female victims by nine to one, and the majority of injuries occurred accidentally. Although the specific demographic information about the gunman was not available, half of all injures were inflicted by friends or relatives, a further $10 \%$ were self-inflicted, and the vast majority occurred in the absence of adult supervision.

It is an offence to discharge any firearm within 50 feet of the centre of a highway (roads, bridle-paths, or footpaths), and therefore particularly concerning that 43 injuries occurred in a public place, two at schools and that 19 were deliberate acts of violence. Air gun crime has become a significant public safety issue with $4 \%$ of people surveyed in the British Crime Survey 2003/4 reporting antisocial behaviour of people with air guns as being a problem in their area. ${ }^{21}$

Most ophthalmologists are unlikely to be familiar with the large number and variety of air guns available and it is unsurprising, therefore, that details relating to the type of weapon involved were variably reported. Despite this, nearly $20 \%$ of injuries involved $0.177 / 0.22$ calibre air guns. However, more than half of all injuries were inflicted by BB guns, which are currently outside Firearms legislation. Whilst BB guns are typically made to look like real hand guns and marketed as toys, it is clear that they are capable of inflicting severe damage to the eye, and must be regarded as a matter for public concern.

The long-term effects of the injuries upon visual function, let alone the resulting social and psychological effects, are distressing and potentially avoidable. Five percent of victims in this study suffered long-term abnormalities of eye movement and $10 \%$ cosmetic defects. Twelve victims lost the injured eye and final vision was hand movements or worse in 26 (19 had no light perception).

This study has shown that all air guns are capable of inflicting severe ocular injury but those involving $0.177 / 0.22$ air guns are more severe and carry a poorer prognosis. Poor presenting visual acuity and severe globe disruption are not unexpectedly associated with a poor final outcome. Although this study focused upon ocular air gun injuries, injuries to the limbs, abdomen, and thorax are twice as common, and Home Office crime statistics are not an accurate reflection of total air gun morbidity.

Recent amendments to the Firearms Act and the Antisocial Behaviour Act 2003 have merely increased by 3 years, the existing law such that the age limits for purchase and possession of air weapons and ammunition is now 17 years, in line with shotgun and firearm certificates. Fourteen year olds are still permitted to use air guns without supervision, on private premises with the consent of the owner. Legislation still does not go as far as requiring certification nor does it place any responsibility upon the owner for security of their air guns. The reasons why these amendments did not go further must in part be economic as licensing the estimated 4-4.5 million air weapons in the United Kingdom would require significant time and resource. It is unlikely that recent amendments to the law will have a significant bearing upon current air weapon use as it is clear that the previous laws were far short of an effective deterrent and difficult to enforce. ${ }^{19}$ The findings of this first ocular air gun surveillance study are discouraging, predicable and draw into question what previous attempts at legislation/education/publicity have achieved.

\section{Acknowledgements}

I would like to acknowledge the support of those contributing the data and co-ordinating the study; $\mathrm{Mr}$ RW Allchin, Mr A Asghar, Mr S Ataullah, Mr HF Bacon, Dr T Barrie, Mr RM Best, Mr S Biswas, Mr RC Bosanquet, Mr GP Brittain, Miss L Butler, Mr AG Casswell, Mr JTK Chan, Mr HC Chen, Mr KG Davey, Mrs S Dhar-munshi, Dr L Esakowitz, Mr TJ Fetherston, Mr JT Gillow, Lt Col MFP Griffiths, Mr K Hakin, Mr EG Hale, Ms J HowardGriffin, Mr DV Inglesbury, Mr NC Kaushik, Mr J KeastBulter, Mr MJ Lavin, Dr DC Manfield, Mr LH Morgan, Dr RI Murray, Mr NU Nabi, Mr NP O'Donnell, Miss FM O'Sullivan, Mr NJ Price, Mr AR Raghu Ram, Mr A Rahman, Mrs A Reynolds, Mr P Riordan-Eva, Mr NJC Sarkies, Mr BR Shrestha, Mr SJ Talks, Mr SA Tarin, Mr AB Tullo, Mrs Madeline Austin, and Mr B Foot.

This study was supported by the Charitable Trusts for the United Bristol Hospitals' (Reg Charity no. 229945) Medical Research Committee, UBHT Headquarters, Marlborough Street, Bristol, BS1 3 NU.

\section{References}

1 Campbell-Hewson G, Egleston CV, Busuittil A. The use of air-weapons in attempted suicide. Injury 1997; 28: 153-158. 
2 Javitt JC, Brenner MH, Curbow B, Legro MW, Street DA. Outcomes of cataract surgery. Improvement in visual acuity and subjective visual function after surgery in the first, second, and both eyes. Arch Ophthalmol 1993; 111: 686-691.

3 Williams C, Harrad RA, Harvey I, Sparrow JM, ALSPAC Study Team. Screening for amblyopia in preschool children: results of a population-based, randomised controlled trial. ALSPAC Study Team. Avon Longitudinal Study of Pregnancy and Childhood. Ophthalmic Epidemiol 2001; 8(5): 279-295.

4 Chua B, Mitchell P. Consequences of amblyopia on education, occupation, and long term vision loss. Br J Ophthalmol 2004; 88(9): 1119-1121.

5 Shuttleworth GN, Galloway PH. Ocular air-gun injuries: 19 cases. J R Soc Med 2001; 94: 1-4.

6 Bratton SL, Dowd MD, Brogan TV, Hegenbarth MA. Serious and fatal air gun injuries: more than meets the eye. Pediatrics 1997; 100: 609-612.

7 Schein OD, Enger C, Tielsch JM. The context and consequences of ocular injuries from air guns. Am J Ophthalmol 1994; 117: 501-506.

8 Sharif KW, McGhee CNJ, Tomlinson RC. Ocular trauma caused by airgun pellets: a ten year survey. Eye 1990; 4: 855-860.

9 Patel BCK. Penetrating eye injuries. Arch Dis Child 1989; 64: 317-320.

10 Jacobs NA, Morgan LH. On the management of retained airgun pellets: a survey of 11 orbital cases. Br J Ophthalmol 1988; 72: 97-100.
11 LaRoche GR, McIntyre L, Schertzer RM. Epidemiology of severe eye injuries in childhood. Ophthalmol 1988; 95: 1603-1607.

12 Moore AT, McCartney A, Cooling RJ. Ocular injuries associated with the use of airguns. Eye 1987; 1: 422-429.

13 Young DW, Little JM. Pellet-gun eye injuries. Can J Ophthalmol 1985; 20: 9-10.

14 Sternberg P, de Juan E, Green WR, Hirst LW, Sommer A. Ocular BB injuries. Ophthalmol 1984; 91: 1269-1277.

15 Bowen DI, Magauran DM. Ocular Injuries cause by airgun pellets: an analysis of 105 cases. BMJ 1973; 1: 333-337.

16 Kreshon MJ. Eye injuries due to BB-guns. Am J Ophthalmol 1964; 58: 858-861.

17 Foot B, Stanford M, Rahi J, Thompson J. The British Ophthalmological Surveillance Unit: an evaluation of the first 3 years. Eye 2003; 17(1): 9-15.

18 Scribano PV, Nance M, Reilly P, Sing RF, Selbst SM. Pediatric nonpowder firearm injuries: outcomes in an urban pediatric setting. Pediatrics 1997; 100(4): E5.

19 HOSB 02/05 February 2005. Crime in England and Wales 2003/2004: supplementary volume 1: Homicide and Gun Crime (edis: David Povey).

20 HOSB 01/04 January 2004. Crime in England and Wales 2002/2003: supplementary volume 1: Homicide and Gun Crime (Eds: David Povey).

21 Perceptions and experience of antisocial behaviour: findings from the 2003/2004 Bristish crime Survey. Martin wood. Home Office Online Report 49/04. 